in vivo $32: 79-83(2018)$

doi:10.21873/invivo.11207

\title{
Evaluation of Vascular Proliferation in Molecular Subtypes of Breast Cancer
}

\author{
IONELA SEVILLA BUJOR ${ }^{1}$, ANDREEA CIOCA ${ }^{1}$, RALUCA AMALIA CEAUȘU ${ }^{1}$, \\ FULGA VEACESLAV $^{2}$, CRISTIAN NICA ${ }^{3}$, ANCA MARIA CIMMPEAN ${ }^{1}$ and MARIUS RAICA ${ }^{1}$ \\ ${ }^{1}$ Department of Histology, Angiogenesis Research Center, ${ }^{3}$ Department of Surgery, \\ Victor Babes University of Medicine and Pharmacy, Timisoara, Romania; \\ ${ }^{2}$ Department of Histology, Cytology and Embryology, \\ Nicolae Testemitanu University of Medicine and Pharmacy, Chisinau, Republic of Moldova
}

\begin{abstract}
Background: Angiogenesis plays a pivotal role in tumor development. Although microvessel density (MVD) is the most common method used for evaluation of angiogenesis, it has several limitations. Our aim was to evaluate MVD and microvessel proliferation (MVP) in a series of invasive breast carcinomas and analyze whether angiogenesis is influenced by the molecular phenotype of each tumor. Materials and Methods: We examined vascular proliferation using double immunohistochemistry (CD34/Ki67) in a series of 54 invasive breast carcinomas and compared the results with standard MVD, molecular subtypes and other classical parameters. Results: Increased MVD and MVP values were recorded in basal-like subtype, but only the MVP value reached significance among this group of patients $(p=0.0001)$. For all cases combined, increased MVP was significantly correlated with negative estrogen receptor $(E R)$ status $(p=0.010)$ and higher histological grade $(p=0.002)$. Conclusion: MVP more accurately reflects the state of angiogenesis in breast cancer, compared with standard MVD. Vascular proliferation was associated with aggressive tumor features, indicating its contribution to tumor progression. The strong association between vascular proliferation and basal-like tumors suggests that this marker can be used for stratification of patients who might benefit from therapies targeting angiogenesis.
\end{abstract}

This article is freely accessible online.

Correspondence to: Assistant Professor Andreea Cioca MD, Ph.D., Department of Microscopic Morphology/Histology, Angiogenesis Research Center, Victor Babes University of Medicine and Pharmacy, Piata Eftimie Murgu 2, 300041 Timisoara, Romania. Tel: +40 722244330, e-mail: cioca_andre@yahoo.com

Key Words: Breast cancer, microvessels, vascular proliferation, immunohistochemistry, angiogenesis.
Angiogenesis is considered a hallmark of cancer and a key requisite in their growth, invasion and progression (1). In 1971, Folkman suggested that tumors can be treated by inhibiting their vascularization (2). It is well known that tumors cannot exceed 2-3 mm without vascular support (2), thus, anti-angiogenic therapy is an attractive target for angiogenesis-dependent tumors such as breast cancer.

Microvessel density (MVD) is the most widely method used for evaluation of angiogenesis, based on counting the vessels in the most vascularized areas of the tumors, namely 'hot spots'. This method was developed by Weidner and coworkers in 1991, who demonstrated that MVD influences the prognostic of patients with breast cancer $(3,4)$. Since then, many other researchers have investigated the role of MVD in breast tumors, but the results are contradictory. However, MVD has some limitations, as it cannot predict the response to therapy or the treatment efficacy (5).

Recent studies showed that microvessel proliferation (MVP), defined as the average number of vessels exhibiting co-expression of an endothelial and a proliferation marker, is a better indicator of angiogenesis compared with MVD (57). In prostate and endometrial carcinomas, microvessel proliferation was found to be a more reliable prognostic marker compared with standard MVD (5-7).

With this background, the aim of the present study was to evaluate vascular proliferation (CD34/Ki67 co-expression) and standard MVD in a series of invasive breast carcinomas, in accordance with the molecular classification. The results were compared by classical clincopathological parameters.

\section{Materials and Methods}

The present study included 54 female patients, aged between 39-85 years (mean $=57.3$ years), who underwent radical modified mastectomy and lymph node dissection between 2009-2013.

Surgical specimens were fixed in buffer formalin and paraffin embedded and $5 \mu \mathrm{m}$-thick step sections were performed for each 
case. The cases were graded according to the WHO Classification of Tumours of the Breast (8) and the Nottingham Grading System (9). Based on conventional histopathological examination, all cases included in this study were diagnosed as ductal invasive carcinomas of no special type (NST) type.

All procedures were carried out according to the principles of the Declaration of Helsinki and were approved by International Review Board of Victor Babeș University of Medicine and Pharmacy, Timișoara, Romania.

Immunohistochemical procedure. For immunohistochemical staining, we selected one representative slide from each case. The technique included heat-induced epitope retrieval with Bond Epitope Retrieval Solution 2, ready-to-use (Leica Biosystems, Newcastle Ltd, Newcastle Upon Tyne, UK) for $20 \mathrm{~min}$. The immunohistochemical technique continued with the blocking of endogenous peroxidases using 3\% hydrogen peroxide for $5 \mathrm{~min}$. Sections were then incubation for $20 \mathrm{~min}$ with primary antibodies to: ER (clone 6F11, ready-to-use), progesterone receptor (PR; clone PGR 323, ready-to-use), human epidermal growth factor receptor 2 (HER2); clone CB11, ready-to-use), Ki67 (clone MIB-1, ready-touse) and cytokeratin 5 (CK5; clone XM26, ready-to-use) all from Novocastra (Leica Biosystems, Newcastle Ltd, Newcastle Upon Tyne, UK). Bond Polymer Refine Detection System (Leica Biosystems) was used for visualization. 3,3 Diamino-benzidine dyhidrochloride was applied as chromogen and hematoxylin was used for counterstaining.

Using immunohistochemistry, the cases were reclassified into four molecular subtypes as follows: $\mathrm{ER}^{+}, \mathrm{PR}^{+}, \mathrm{HER} 2^{-}, \mathrm{CK} 5^{-}$and $\mathrm{Ki} 67<14 \%$ as luminal A; $\mathrm{ER}^{+}$with/without $\mathrm{PR}^{+}, \mathrm{HER}^{+}$, $\mathrm{CK}^{-}$or $\mathrm{ER}^{+}$with/without $\mathrm{PR}^{+}, \mathrm{HER} 2^{-}, \mathrm{CK}^{-}$and $\mathrm{Ki} 67>14 \%$ as luminal B; $\mathrm{ER}^{-}, \mathrm{PR}^{-}, \mathrm{HER} 2^{+}, \mathrm{CK}^{-}$as HER2-overexpressing; ER ${ }^{-}, \mathrm{PR}^{-}$, HER2 ${ }^{-}$and $\mathrm{CK}^{+}{ }^{+}$as triple-negative/basal-like (10). ER and PR were scored accordingly to the Allred system (11), and HER2 accordingly to American Society of Clinical Oncology recommendations (12). For the Ki-67 proliferation index we used a 14\% threshold as the limit to define high/low proliferative cases (10).

Immunohistochemical study included double staining with CD34/Ki67. Heat-induced epitope retrieval with $\mathrm{pH} 6.0$ solution (Leica Biosystems) for 30 minutes was followed by endogenous peroxidase blocking ( $3 \%$ hydrogen peroxide, $5 \mathrm{~min}$ ). The procedure continued with incubation with primary antibody Ki67 (clone MIB1, ready-to-use, 30 minutes; Novocastra), and then with the second antibody to CD34 (clone Qbend10, ready-to-use, $30 \mathrm{~min}$; Novocastra), visualized with Warp Red as chromogen, for $10 \mathrm{~min}$ (Biocare Medical, LLC, Concord, CA, USA). The procedure was performed with Bond Refine Detection System DAB/RED.

Assessment of neovascularization. The hot-spot method was applied for MVD assessment (13). Therefore, the most vascularized areas of the tumor were selected and both proliferative and nonproliferative blood vessels were counted at $\times 400$ magnification. Proliferating microvessels were counted in the same fields as MVD, at $\times 400$ magnification and were defined as cells lining the vessel lumen that expressed both CD34 (red staining) and Ki67 (brown staining). Proliferative vessels were counted in a semi-automated manner using the method previously described by Suciu et al. (14).

Image acquisition and analysis were performed using a Nikon Eclipse E 600 microscope (Nikon Microscopes/Instruments Division, Vienna, Austria) and Lucia G software (Laboratory
Table I. Characteristics of the patients for each molecular subtype of breast cancer.

\begin{tabular}{|c|c|c|c|c|c|}
\hline Characteristic & LA & LB & HER2 & BL & Total \\
\hline No. of cases & 32 & 11 & 2 & 9 & 54 \\
\hline \multicolumn{6}{|l|}{ Age, years } \\
\hline Median & 55.2 & 57.0 & 67.0 & 72.4 & 57.3 \\
\hline \multicolumn{6}{|c|}{ Tumor diameter, $\mathrm{n}$} \\
\hline$<2 \mathrm{~cm}$ & 6 & 4 & 0 & 4 & 14 \\
\hline$\geq 2 \mathrm{~cm}$ & 26 & 7 & 2 & 5 & 40 \\
\hline \multicolumn{6}{|l|}{ Grade, $\mathrm{n}$} \\
\hline 1 & 2 & 0 & 1 & 0 & 3 \\
\hline 2 & 17 & 7 & 1 & 4 & 29 \\
\hline 3 & 13 & 4 & 0 & 5 & 22 \\
\hline \multicolumn{6}{|l|}{ Stage, $\mathrm{n}$} \\
\hline I & 6 & 4 & 0 & 4 & 14 \\
\hline II & 21 & 5 & 0 & 5 & 31 \\
\hline III, IV & 5 & 2 & 2 & 0 & 9 \\
\hline \multicolumn{6}{|c|}{ Lymph node status, $\mathrm{n}$} \\
\hline Negative & 28 & 9 & 1 & 5 & 44 \\
\hline Positive & 3 & 2 & 1 & 4 & 10 \\
\hline \multicolumn{6}{|l|}{ Ki67 index, n } \\
\hline High $(>15 \%)$ & 1 & 8 & 2 & 8 & 19 \\
\hline Low $(<15 \%)$ & 30 & 3 & 0 & 1 & 34 \\
\hline \multicolumn{6}{|l|}{ MVD } \\
\hline Median & 19 & 23.4 & 20.6 & 25.3 & 21.7 \\
\hline \multicolumn{6}{|l|}{ MVP } \\
\hline Median & 2.1 & 3.1 & 3 & 3.5 & 2.9 \\
\hline \multicolumn{6}{|l|}{ ER status, $\mathrm{n}$} \\
\hline Positive & 32 & 11 & 0 & 0 & 43 \\
\hline Negative & 0 & 0 & 2 & 9 & 11 \\
\hline \multicolumn{6}{|l|}{ PR status, $n$} \\
\hline Positive & 31 & 8 & 0 & 0 & 39 \\
\hline Negative & 1 & 3 & 2 & 9 & 15 \\
\hline
\end{tabular}

MVD: Microvessel density; MVP: microvessel proliferation; ER: estrogen receptor; PR: progesterone receptor; LA: luminal A; LB: luminal B; HER2: human epidermal growth factor receptor 2overexpressing; BL: basal-like.

Imaging, Prague, Czech Republic) for microscopic image analysis. The entire immunohistochemical procedure was performed with Leica Bond Max (Leica Biosystems) autostainer.

Statistical methods. To assess the relationship between clinicopathological parameters and the immunohistochemical markers, we used Pearson, Spearman and Student $t$-test. $p$-Values of less than 0.05 were considered statistically significant. All statistical analysis was performed using the commercially available SPSS 22.0 software for Windows (IBM Corp., Armonk, NY, USA).

\section{Results}

All cases included in the present study were histopathologically diagnosed as invasive ductal carcinoma of NST type. Most cases were graded as G2 (29 cases, 54\%), followed by grade 3 (22 cases, 41\%), while only three 

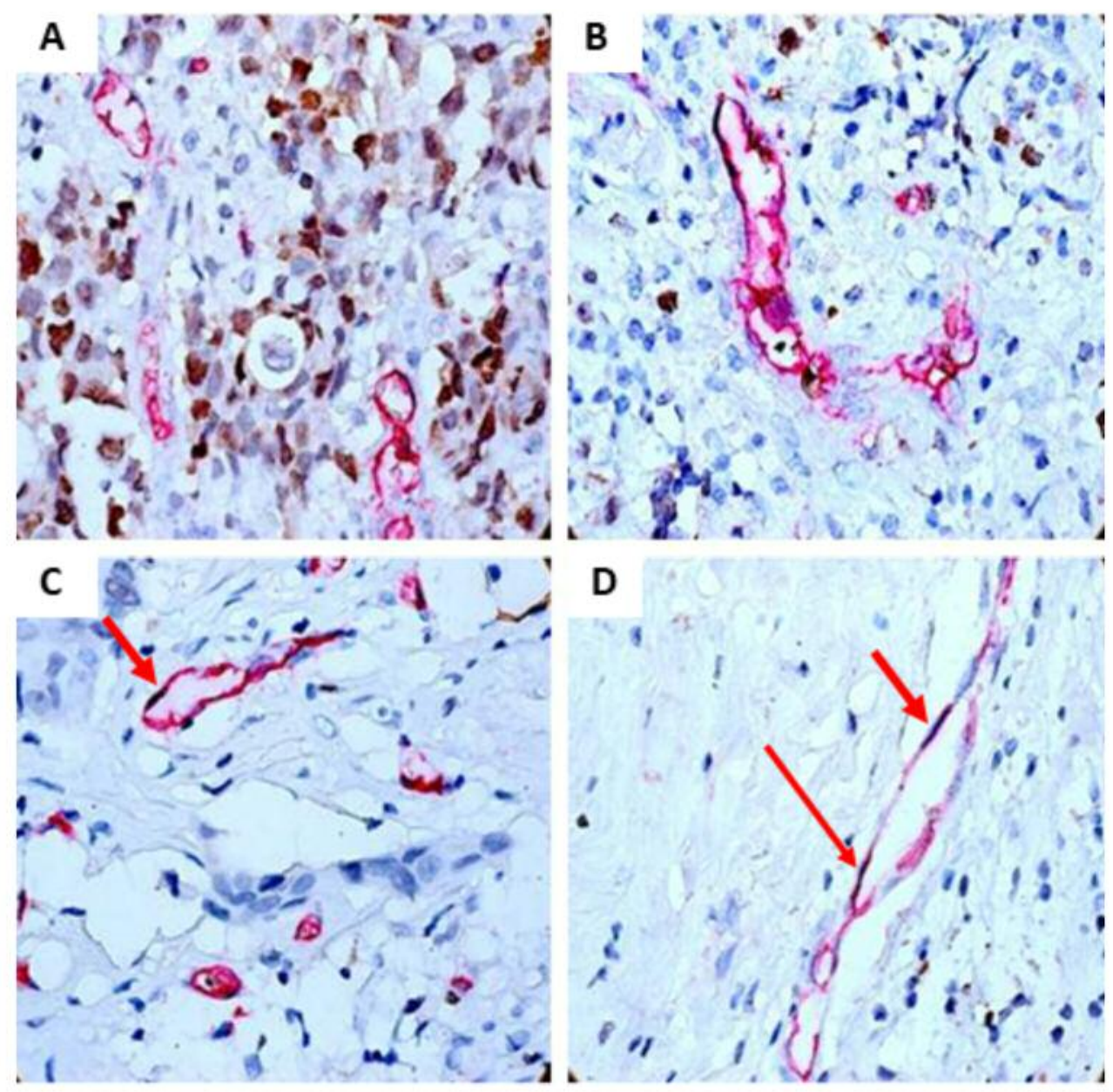

Figure 1. Angiogenesis in invasive breast carcinoma indicated by double immunohistochemistry (CD34/Ki67) showing small blood vessels (red) accompanied by endothelial cells (brown) (A, B), and small blood vessels with lumen lined by proliferating endothelial cells (arrow) (C), as well as vascular structures with a 'cord-like' aspect with proliferating endothelial cells (arrows) (D); (magnification $\times 400)$.

tumors were graded as G1 $(5 \%)$. The median age at diagnosis was 57.3 years (range $=39-85$ years). Of the 54 cases included in our study, 40 tumors $(74 \%)$ were larger than $2 \mathrm{~cm}$, while 14 tumors $(26 \%)$ were smaller than $2 \mathrm{~cm}$. The median tumor diameter was $5.8 \mathrm{~cm}$ (range $=1.3-12 \mathrm{~cm}$ ).

By using the molecular surrogate markers, 32 out of 54 $(59 \%)$ cases were classified as luminal A subtype, 11 cases (20\%) were luminal B, two cases (4\%) were HER2overexpressing and nine cases $(17 \%)$ had a basal-like profile. Characteristics of the patients for each molecular subtype are illustrated in Table I.

For all tumors combined, the median MVD was 21.76 vessels (range $=6-68$ ), while median MVP was 2.92 vessels (range=1-16). When each molecular subtype was evaluated separately, the highest median MVD and MVP values were recorded in the basal-like group (25.3 vessels and 3.5 vessels, respectively), while the lowest values were in luminal A tumors (19 vessels and 2.1 vessels, respectively). Notably, only MVP value reached significance in this group of patients $(p=0.0001)$. The highly angiogenic process that characterized basal-like tumors was sustained by the presence of small blood vessels accompanied by many endothelial cells (Figure 1A). In contrast, the other molecular subtypes were characterized by vessels with a larger lumen and with isolated endothelial cells (Figure 1B). All molecular subtypes not only contained small blood vessels with a lumen lined by proliferating endothelial cells, but also had vascular structures that tended to form a lumen, with a 'cordlike' aspect (Figure 1C and D).

For all cases combined, increased MVP was significantly correlated with negative ER status $(p=0.010)$ and with higher histological grade $(p=0.002)$. However, these associations 
were not found when cases were analyzed separately based on molecular subtypes.

Neither MVD nor MVP showed any association with the other clinicopathological parameters included in this study; moreover, no correlation was found between MVD and MVP.

\section{Discussion}

It is already known that angiogenesis has a direct impact on breast cancer development and that its level influences the prognostis of patients with this malignancy $(3,15,16)$. Although the targets of anti-angiogenic agents are rational, these agents failed to add significant clinical benefits or to improve survival in patients with breast cancer (1). A possible explanation is that these anti-angiogenic agents were tested in unselected patients, without taking into account their molecular profile (1).

The concept of 'heterogeneity' in breast cancer is now widely accepted. With the advancements of new molecular techniques, it has been demonstrated that breast cancer is not a single disease but a heterogeneous one that comprise various molecular phenotypes characterized by specific behavior and prognosis (17). Molecular classification divides patients into subgroups based on their various gene expression including luminal A, luminal B, HER2 and basallike tumors (17). This classification not only has a valuable contribution to the management of patients with this malignancy but also represents a step forward in the new era of personalized therapy.

Previous studies showed that vascular proliferation is a sensitive method for evaluation of angiogenesis, with more reliable results compared with standard MVD (18-21). MVP is a relatively new parameter of angiogenesis that measures the most active tumor vasculature, while MVD includes both preformed and newly formed vessels $(6,7)$. Thus, vascular proliferation reflects ongoing angiogenesis by reducing the possibility of counting vessels that are not produced by the tumor (18).

In the present study, we evaluated the relationship between angiogenesis and the molecular subtypes of invasive breast carcinoma in a series of 54 patients. We showed that basal-like tumors were associated with increased angiogenesis as estimated by MVD and MVP. However, only MVP reached significance among this group of patients $(p=0.0001)$. Our results are in line with previous reports that found increased vascular proliferation in basal-like breast tumors, but using different markers of vascular proliferation (18-20). The mechanism of this association is not known, although some authors suggest that vascular endothelial growth factor (VEGF), as a regulator of angiogenesis, contributes to increased vascular proliferation in basal-like tumors $(18,22)$. Basal-like tumors are associated with a high rate of hematogenous metastasis and with the poorest prognosis among all molecular groups. Based on our results, we suggest that vascular proliferation can be used for stratification of patients who might benefit from therapies targeting angiogenesis.

When analyzing all the cases in this study, we found that increased MVP was significantly correlated with both negative ER status $(p=0.010)$ and high histological grade $(p=0.002)$, suggesting that vascular proliferation has a significant role in breast cancer progression. However, these associations were not found when cases were analyzed separately based on their molecular subtypes. Arnes and coworkers studied a large cohort of patients with breast cancer and demonstrated that vascular proliferation is an important prognostic factor in high-grade and ER-negative breast tumors. By contrast, MVD was not a significant prognostic indicator in their series of breast cancers, which our results are comparable with (5).

In conclusion, our study demonstrated that quantification of vessels with proliferating endothelium more accurately reflects the state of angiogenesis in breast cancer compared with standard MVD. In addition, we showed that vascular proliferation is associated with aggressive tumor features, indicating its contribution to breast cancer progression. The strong association between vascular proliferation and basallike tumors suggests that this MVP might have an important contribution to the management of this particular group of patients. However, further studies are required to fully understand the mechanism of angiogenesis in relation to basal-like tumors and to validate the role of MVP in stratification of patients who might benefit from antiangiogenic therapies.

\section{Acknowledgements}

The present work was supported by the Internal Research Program, PII-C5-TC-2017 (ANGIOLIMFMETAMAM), granted by Victor Babes University of Medicine and Pharmacy, Romania.

\section{References}

1 Aalders KC, Tryfonidis K, Senkus E and Cardoso F: Antiangiogenic treatment in breast cancer: Facts, successes, failures and future perspectives. Cancer Treat Rev 53: 98-110, 2017.

2 Folkman J: Tumor angiogenesis: therapeutic implications. N Engl J Med 285: 1182-1186, 1971.

3 Weidner N, Semple JP, Welch WR and Folkman J: Tumor angiogenesis and metastasis - correlation in invasive breast carcinoma. N Engl J Med 324: 1-8,1991.

4 Weidner N, Folkman J, Pozza F, Bevilacqua P, Allred EN, Moore DH, Meli S and Gasparini G: Tumor angiogenesis: a new significant and independent prognostic indicator in early-stage breast carcinoma. J Natl Cancer Inst 84: 1875-1887, 1992.

5 Arnes JB, Stefansson IM, Straume O, Baak JP, Lønning PE, Foulkes WD and Akslen LA: Vascular proliferation is a prognostic factor in breast cancer. Breast Cancer Res Treat 133: 501-510, 2012. 
6 Stefansson IM, Salvesen HB and Akslen LA: Vascular proliferation is important for clinical progress of endometrial cancer. Cancer Res 66: 3303-3309, 2006.

7 Gravdal K, Halvorsen OJ, Haukaas SA and Akslen LA: Proliferation of immature tumor vessels is a novel marker of clinical progression in prostate cancer. Cancer Res 69: 47084715, 2009.

8 Colditz G and Chia KS: Invasive breast carcinoma: Introduction and general features. In: World Health Organization Classification of Tumours of the Breast. Lakhani SR, Ellis IO, Schnitt SJ, Tan PH and van de Vijver MJ (eds.). International Agency for Research on Cancer (IARC), Lyon, pp. 19-20, 2012.

9 Elston CW and Ellis IO: Pathological prognostic factors in breast cancer. I. The value of histological grade in breast cancer: experience from a large study with long-term follow-up. Histopathology 19: 403-410, 1991.

10 Fulga V, Rudico L, Balica AR, Cimpean AM, Saptefrati L, Margan MM and Raica M: Differential expression of e-cadherin in primary breast cancer and corresponding lymph node metastases. Anticancer Res 35: 759-765, 2015.

11 Allred DC, Harvey JM, Berardo M and Clark GM: Prognostic and predictive factors in breast cancer by immunohistochemical analysis. Mod Pathol 11: 155-168, 1998.

12 Wolff AC, Hammond ME, Hicks DG, Dowsett M, McShane LM, Allison KH, Allred DC, Bartlett JM, Bilous M, Fitzgibbons P, Hanna W, Jenkins RB, Mangu PB, Paik S, Perez EA, Press MF, Spears PA, Vance GH, Viale $G$ and Hayes DF: Recommendations for human epidermal growth factor receptor 2 testing in breast cancer: American Society of Clinical Oncology/College of American Pathologists Clinical Practice Guideline Update. J Clin Oncol 31: 3997-4013, 2013.

13 Vladau M, Cimpean AM, Balica RA, Jitariu AA, Popovici RA and Raica M: VEGF/VEGFR2 axis in periodontal disease progression and angiogenesis: basic approach for a new therapeutic strategy. In Vivo 30: 53-60, 2016.

14 Suciu C, Muresan AM, Cornea R, Suciu O, Dema A and Raica M: Semi automated evaluation of Ki67 index in invasive ductal carcinoma of the breast. Oncol Lett 7: 107-114, 2014.

15 Horak ER, Klenk N, Leek R, LeJeune S, Smith K, Stuart N, Greenall M, Stepniewska K and Harris AL: Angiogenesis, assessed by platelet/endothelial cell adhesion molecule antibodies, as indicator of node metastases and survival in breast cancer. Lancet 340: 1120-1124, 1992.
16 Vartanian RK and Weidner N: Correlation of intratumoral endothelial cell proliferation with microvessel density (tumor angiogenesis) and tumor cell proliferation in breast carcinoma. Am J Pathol 144: 1188-1194, 1994.

17 Eliyatkın N, Yalçın E, Zengel B, Aktaş S and Vardar E: Molecular classification of breast carcinoma: from traditional, old-fashioned way to a new age, and a new way. The J Breast Health 11: 59-66, 2015.

18 Nalwoga H, Arnes JB, Stefansson IM, Wabinga H, Foulkes WD and Akslen LA: Vascular proliferation is increased in basal-like breast cancer. Breast Cancer Res Treat 130:1063-1071, 2011.

19 Foulkes WD, Brunet JS, Stefansson IM, Straume O, Chappuis PO, Begin LR, Hamel N, Goffin JR, Wong N, Trudel M, Kapusta L, Porter P and Akslen LA: The prognostic implication of the basal-like (cyclin E high/p27 low/p53?/glomeruloidmicrovascular-proliferation?) phenotype of BRCA1-related breast cancer. Cancer Res 64:830-835, 2004.

20 Krüger K, Stefansson IM, Collett K, Arnes JB, Aas T and Akslen LA: Microvessel proliferation by co-expression of endothelial nestin and $\mathrm{Ki}-67$ is associated with a basal-like phenotype and aggressive features in breast cancer. Breast 22:282-288, 2013.

21 Bosari S, Lee AK, DeLellis RA, Wiley BD, Heatley GJ and Silverman ML: Microvessel quantitation and prognosis in invasive breast carcinoma. Hum Pathol 23:755-761, 1992.

22 Ribeiro-Silva A, Ribeiro do Vale F and Zucoloto S: Vascular endothelial growth factor expression in the basal subtype of breast carcinoma. Am J Clin Pathol 125:512-518, 2006.
Received September 17, 2017

Revised October 7, 2017

Accepted October 13, 2017 